\title{
Paediatric abdominal trauma
}

Mayday University Hospital, London Road, Thornton Heath CR7 7YE, UK:

Department of Urology

J Shah

Department of General Surgery $S$ B Middleton J Derodra

Correspondence to: Miss Jyoti Shah, Department of Urology, St Mary's Hospital, Praed Street, London W2 1NY, UK jyoti.shah@ic.ac.uk

Submitted 21 August 2000 Accepted 7 November 2000

\section{J Shah, S B Middleton, J Derodra}

A 7 year old boy presented to the accident and emergency department with severe, diffuse abdominal pain 30 minutes after a road traffic accident. He had been the only passenger wearing a seat belt in the car, which had been involved in a head-on collision.

On admission he was alert with a pulse rate of 90 beats/min, respiratory rate of 30 breaths/ min, and a blood pressure of 135/85 mm Hg. Auscultation of the chest was unremarkable and examination of his abdomen revealed diffuse tenderness, guarding, and absent bowel sounds. There was no blood at the anus or the external urethral meatus. An erect chest radiograph did not reveal any free intraperitoneal gas. Computed tomography of his abdomen is shown in fig 1 .

\section{Questions}

(1) What does the computed tomogram show?

(2) How should this child be managed?

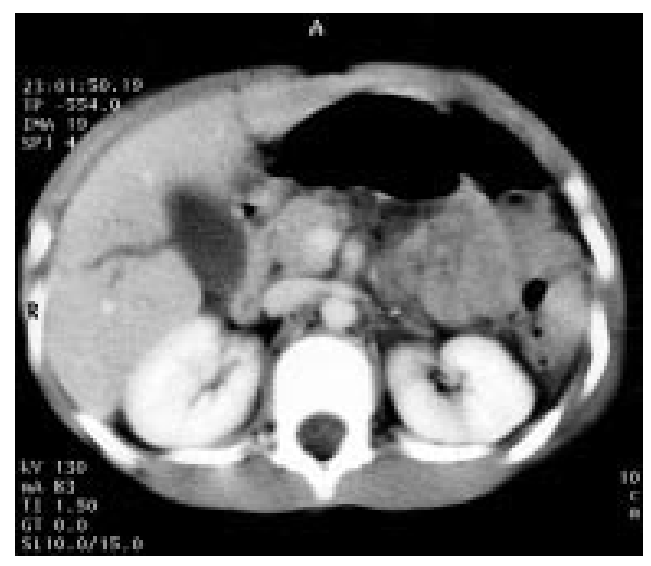

Figure 1 Computed tomogram of the abdomen. 
Answers

QUESTION 1

The computed tomogram of this child's abdomen shows transection of the posterior part of the right lobe of the liver.

QUESTION 2

A high index of suspicion for liver trauma must be maintained when evaluating children who have sustained blunt abdominal trauma. Initial resuscitation of such a case of trauma follows basic principles and includes management of the airway, breathing, and circulation. Once oxygen is being delivered via an unobstructed airway and the child is breathing, circulation is the next priority. Immediate intravenous access should be obtained with two large bore cannulae. In the event that this is not possible, the interosseous route is both effective and useful in children.

Twenty five per cent of a child's circulating blood volume may be lost without any reduction in arterial blood pressure and so this is an inaccurate form of assessment of hypovolaemia. More subtle signs of circulatory failure such as changes in skin turgor, cooling of the extremities, and abnormal lethargy are often more helpful.

Fluid resuscitation should be started with an intravenous bolus of $20 \mathrm{ml} / \mathrm{kg}$ of either a colloid such as $4.5 \%$ human albumin solution or of a crystalloid such as normal (physiological) saline. ${ }^{1}$ A further bolus of $20 \mathrm{ml} / \mathrm{kg}$ is repeated if further assessment demonstrates no improvement in the child's vital signs. A maximum of two such boluses may be given. If the child is still haemodynamically unstable after this, intravenous blood should be administered. Cross matched blood may take as long as one hour to become available and if blood is required in an emergency, then either noncross matched type specific blood, which can be prepared in 15 minutes, or $\mathrm{O}$ negative blood should be given.

A full blood count, urea and electrolytes, liver function tests, serum amylase, and cross match should be requested early. Urinalysis should also be performed. An abdominal radiograph and erect chest radiograph may show evidence of free intraperitoneal gas or organ displacement. Vital signs should be continuously monitored, and when the patient is stable computed tomography should be performed.

Once the diagnosis is established, the patient must be transferred to a regional paediatric unit. The patient should be continuously re-evaluated. In this case, the patient remained stable. No further action was taken and he was managed conservatively. $\mathrm{He}$ underwent serial computed tomography and was asymptomatic when discharged three weeks later.

\section{Conservative $v$ surgical management}

Urgent surgical intervention is essential if the patient fails to respond to resuscitation, or if the clinical condition subsequently deteriorates. The aims of surgery are to control haemorrhage, debride devitalised tissues, and to drain perihepatic spaces.
The complications of surgery are haemorrhage, biliary leak, or a loss of the tamponade effect of non-intervention, potentially leading to torrential haemorrhage. In addition, introduction of bacteria leads to the possibility of seeding of hepatic collections. These are significant complications and the finding that up to $45 \%$ of patients explored surgically after blunt liver trauma required only drainage of non-bleeding lacerations ${ }^{2}$ has lead to a tendency to treat such patients conservatively. On the other hand, conservative management risks persistent haemorrhage and massive transfusion requirements, sepsis, delayed rupture, haemobilia, and consequently prolonged hospital stay. Nevertheless, conservative management is successful in up to $97 \%$ of cases. ${ }^{3}$

\section{Imaging in blunt abdominal trauma}

Angiography may localise active bleeding and guide subsequent therapeutic embolisation but it is invasive and a high complication rate in children precludes its routine use in acute liver trauma. Ultrasonography is cheap and avoids exposure to radiation but technical problems such as the presence of gas and a requirement for deep inspiration, which may be impossible, lessen its efficacy. In addition it does not adequately assess the superior hepatic parenchyma and is unable to provide functional information regarding hepatic perfusion. ${ }^{4}$

Computed tomography remains the single best imaging method in acute liver trauma in children. ${ }^{5}$ Despite its cost, radiation exposure, and a need for the child to be immobile, perhaps necessitating sedation, it is invaluable in demonstrating the nature, segmental distribution, and severity of liver damage as well as the relationship of the injury to major blood vessels. It can identify coexistent injury to other intra-abdominal organs and allows a decision to be made regarding conservative management or operative intervention, obviating the need for diagnostic peritoneal lavage. Should surgery be necessary computed tomography enables a carefully planned procedure to be performed through an appropriately placed incision. In the longer term management of blunt liver trauma, computed tomography enables re-evaluation of hepatic injuries and may guide radiological drainage of perihepatic collections.

\section{Final diagnosis}

Transection of the posterior part of the right lobe of the liver.

1 Advanced Life Support Group. Advanced paediatric life support: the practical approach. 2nd Ed. London: BMJ Publishing Group, 1997

2 Bass BL, Eichelberger MR, Schisgall R, et al. Hazards of nonoperative therapy of hepatic injury in children. F Trauma 1984;24:978-82.

3 Bond SJ, Eichelbereger MR, Gotschall CS, et al. Nonoperative management of blunt hepatic and splenic injury in children. Ann Surg 1996;223:286-9.

4 Kaufman RA, Towbin R. Babcock DS, et al. Upper abdominal trauma in children. Imaging evaluation. Am f Roentgenol 1984;142:449-60.

5 Vock P, Kehrer B, Tschaeppeler H. Blunt liver trauma in children: the role of computed tomography in diagnosis and treatment. F Pediatr Surg 1986;21:413-18. 